\title{
La caligrafía árabe relatada por Rafik Schami: una propuesta de traducción (alemán- español)
}

\author{
María Pilar Castillo Bernal \\ z92cabep@uco.es \\ Universidad de Córdoba \\ Lourdes Bonhome Pulido \\ 142bopul@uco.es \\ Universidad de Córdoba
}

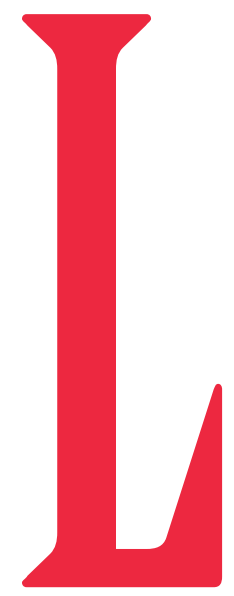

a novela histórica es un género literario que nunca ha perdido seguidores. Por el contrario, en la última década, el interés por esta narrativa ha ido en aumento. Prueba de ello son las numerosas obras -algunas en forma de saga- que toman como eje central para el desarrollo de su trama un acontecimiento histórico o un personaje cuya vida pasó a la historia. Sin embargo, estas obras no siempre son fieles a la realidad de la historia, claramente debido al deseo de sus autores de atraer al público y atraparlo

en su narración.

En el caso de la obra que presentamos hoy, el autor se ha basado para desarrollar su narración en el famoso personaje de Ben Muqla, uno de los calígrafos árabes más importantes para la historia de esta disciplina. La trama que Schami nos propone no tiene a Ben Muqla como personaje de la misma, sino que la relación con el calígrafo es, precisamente, la caligrafía. La repercusión de esta obra en los países de habla alemana ha llevado a que la misma sea traducida a otras lenguas, entre ellas el español. Esta traducción ha sido realizada por Carlos Fortea para la editorial Salamandra y publicada en 2009. Es esta edición la que vamos a emplear para la realización de nuestro trabajo, junto con la novela original de bolsillo en alemán publicada por la editorial dtv en 2012. Así pues, dentro del contexto de la traducción al español de la obra de Schami, proponemos un doble estudio. Por un lado, realizaremos una propuesta de traducción al español del epílogo de la obra, que no ha sido incluido en la versión española de la misma. Por otro lado, presentamos un análisis del lenguaje de la caligrafía árabe desarrollada por Ben Muqla que aparece en dicho epílogo. Para ello, incluimos en nuestro trabajo un glosario terminológico extraído de dicho epílogo en el cual se desarrolla la historia de la caligrafía árabe y, por tanto, encontramos un lenguaje especializado relacionado con este campo semántico.

\section{Raflk Schami: un escritor sirio en Alemania}

Schami nació en Damasco en 1946 y su nombre real es Suheil Fadél. El pseudónimo Rafik Schami significa “amigo damasceno". Emigró a Alemania en 1971 y en 1979 se doctoró en Química, desde entonces ha obtenido la doble nacionalidad y ha publicado unas 72 obras en lengua alemana, según se recoge en la página web oficial del autor. Sus obras han sido traducidas a 30 idiomas y ha recibido unos 34 premios y reconocimientos. Es miembro de la Academia de Bellas Artes de Baviera y cofundador del grupo literario Südwind y de la asociación PoLiKunstVerein, dos grupos interculturales que engloban escritores de distintas nacionalidades "con la finalidad de alcanzar un público amplio y lograr un cambio social y político a través de su literatura" (Nasarre, 2013: 91). Se trata de una nueva generación de escritores nacidos de la emigración, como 


\section{Resumen:}

El presente trabajo tiene por objeto la traducción y el comentario de un relato del autor sirio afincado en Alemania Rafik Schami. El relato, incluido a modo de epílogo de la novela Das Geheimnis des Kalligraphen, no ha sido publicado en español pese al interés de la temática que aborda, centrada en la caligrafía árabe y en la figura del célebre calígrafo Ben Muqla. En esta investigación se realizará una traducción del alemán al español y se revisarán los conceptos claves de los estilos y el lenguaje caligráfico comentados por Schami. Asimismo, las caligrafías en lengua árabe incluidas en el relato serán objeto de análisis en un estudio comparativo con el texto original y el texto meta. Con ello, pretendemos abordar las dificultades de la traducción de conceptos técnicos propios de una tercera lengua, ajena a la combinación en la que se realiza la labor traductora, desde una metodología de trabajo multidisciplinar. Un objetivo secundario de este trabajo es contribuir a la difusión de la obra de Schami en el ámbito español, especialmente en lo que se refiere a sus ideas sobre la lengua árabe y las reformas de su alfabeto.

Palabras dave: Caligrafía, lengua árabe, alemán, traducción literaria.

\section{Abstract:}

IThe purpose of this work is to translate and analyse a short story by Syrian author Rafik Schami, who lives in Germany and writes in German. This story, appended as an epilogue to the novel Das Geheimnis des Kalligraphen, has not been published in Spanish despite the interest of the topic: Arabic calligraphy and the figure of renowned calligrapher Ibn Muqla. The story titled Was ich schaffe, überdauert die Zeit has been translated from German into Spanish and the key concepts of calligraphic styles explained by Schami are reviewed. Furthermore, several calligraphed texts in Arabic included in the story are studied in a comparative analysis with the source and target texts. This work is an interdisciplinary approach to the difficulties of translating technical concepts in a third language other than the language combination of a particular translation. A secondary aim is to contribute to the dissemination of Schami's works in Spain, especially as far as his thoughts on the reforms of the Arabic alphabet are concerned.

Key words: Calligraphy, Arabic, German, literary translation muestra la serie literaria "Südwind-Gastarbeiterdeutsch", coeditada por Schami entre 1980 y 1985.

Además de su compromiso con el diálogo intercultural, cristalizado en la fundación Schams e.V. para el apoyo a los niños y jóvenes sirios en 2012 o en sus esfuerzos por la promoción del diálogo palestino-israelí, la obra de Schami se centra en su tierra natal y su idiosincrasia. Su obra más conocida y que tardó 30 años en escribir, Die dunkle Seite der Liebe (2008), es "un análisis de los linajes árabes y su papel en la conformación del espíritu árabe”, según explica el autor en una entrevista (Corroto, 2009). El secreto del calígrafo, la novela cuyo epílogo analizamos en el presente trabajo, se basa en la experiencia del autor: "Esta novela no necesitó tanto tiempo. Conocía bien el tema porque trabajé tres años junto a un calígrafo. También conozco los problemas del lenguaje árabe que el héroe de esta historia, Hamid Farsi, quiere corregir." (Ibid.) Esta novela también se basa en la cultura de los linajes, ya que relata la relación de una mujer musulmana con un joven cristiano en el Damasco de los años 50 del pasado siglo.

La popularidad de Schami en su país de adopción, Alemania, es considerable y proviene en gran parte del compromiso social del autor y de su presencia en la vida literaria, de la mano de numerosas lecturas públicas y de su presencia en ferias del libro y eventos similares.
Lamentablemente, en nuestro país su obra no goza de una fama equivalente, a pesar de la importancia histórica y social del mundo árabe en el contexto español. Con nuestro estudio, esperamos contribuir a la recepción de la obra de Schami y, más específicamente, de la figura de Ben Muqla y las características de la caligrafía árabe.

\section{Ben Muqla y la caligrafía árabe}

Si existe un personaje relevante para la historia de la caligrafía árabe, este es sin duda Abū 'Alī Muhammad ibn 'Alī ibn Muqla, más conocido como Ben Muqla. Nacido en Bagdad a finales del S. IX, su vida estuvo relacionada con la caligrafía desde muy temprano gracias a la dedicación a esta disciplina de su abuelo y de su padre. Antes de ser calígrafo, Ben Muqla trabajó recaudando impuestos en Fārs, donde accedió al puesto de secretario dentro del gobierno de Ibn al-Furāt, convirtiéndose más tarde en visir del gobierno. Su vida política le hizo ganar muchos amigos, pero también enemigos y, casi al final de su vida, fue llevado a prisión donde murió en el 940.

La labor de Ben Muqla como calígrafo ha pasado a la historia por el desarrollo de la caligrafía que este hizo. Ben Muqla creó seis nuevos estilos caligráficos que pronto fueron acogidos como sistemas de escritura fundamentales en todo 
el territorio musulmán. Su visión de la caligrafía contrasta con las matemáticas y la música. Ben Muqla concebía la escritura como una armonía de formas y trazos, por lo que basó su sistema de caligrafía en la repetición exacta de la medida del alif usando para ello cálculos matemáticos que aseguraban una proporcionalidad exacta entre las letras. Si se tiene en cuenta que la grafía árabe se caracteriza no solo por comenzar su trazo en la derecha, sino también porque

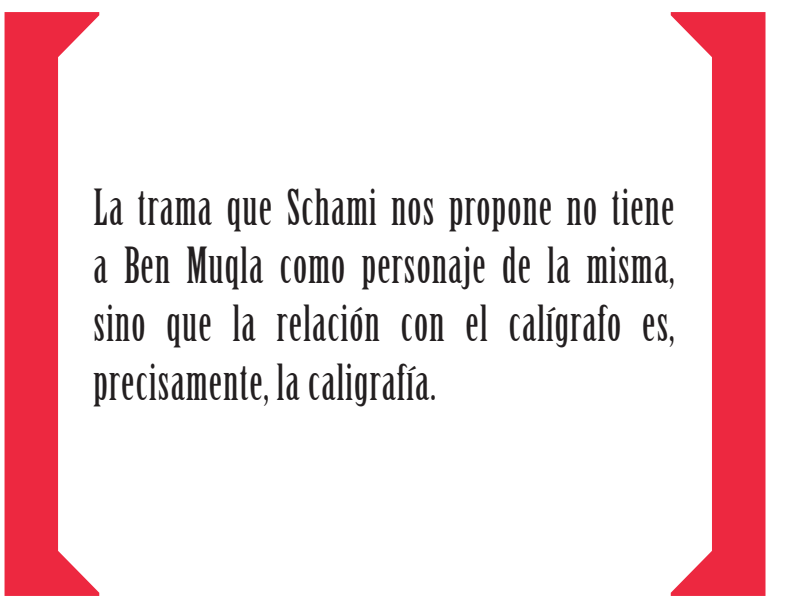

sus letras se unen entre sí (excepto en algunos casos), esto permite que la caligrafía se torne bella según el trazado. Los sistemas de caligrafía por Ben Muqla fueron seis: el naskhī (empleado en el s. XI para escribir el Corán), el farsi, el rihani, el cúfico, el tawqī, el thuluth, el riqa'i y el diwānī.

\section{Was ich schaffe, überdavert die Zeit}

"Lo que yo cree sobrevivirá al tiempo". Estas son las palabras que Ben Muqla hizo grabar en los sillares del muro de su jardín en su palacio de Bagdad y que dan título al relato objeto de nuestro análisis. En el epílogo a El secreto del calígrafo, Schami cuenta "Una historia sobre la belleza de la caligrafía”, como reza el subtítulo, con cinco ilustraciones caligrafiadas a color por Ismat Amiralai. La macroestructura del epílogo consta de cinco apartados, siendo el primero el relato de la vida de Ben Muqla que se incluye en el cuerpo de la novela (Schami 2012: 235-242). Los restantes epígrafes se titulan "Die arabische Schrift. Musik für die Augen", "Schreibstile", "Musik für die Augen" $\mathrm{y}$ "Ein schönes Alphabet mit ein paar Makeln". No ha sido posible establecer el motivo de que la editorial Salamandra no decidiera incluir la traducción del epílogo en la versión española, aunque podría deberse a que el relato que nos ocupa sea de factura posterior y se añadiera con motivo de la publicación de la edición de bolsillo en 2012, con lo que la traducción española de 2009 lógicamente no habría contado con este texto original. Sin embargo, nos parece innegable el interés del mismo en el ámbito español, tan ligado a la cultura árabe y su caligrafía, que puede ser observada hoy día en los numerosos monumentos del periodo andalusí.

\subsection{Los poemas y aforismos}

En lo sucesivo, analizaremos las traducciones propias de los fragmentos no incluidos en la versión española de Carlos Fortea relacionadas con la caligrafía árabe. El relato que cierra la novela contiene diversos aforismos y poemas que imprimen un mayor lirismo al epílogo. Las bellas caligrafías de Amiralai contribuyen al interés del mismo, por lo que las hemos incluido en las figuras 1-5 además de la transcripción de los textos originales en árabe. La metodología seguida en la traducción y el análisis de los fragmentos caligrafiados se basa en la traducción del alemán al español sin comparar el texto original (TO) con la caligrafía y en la posterior corrección o comentario teniendo en cuenta tanto el TO alemán como el texto meta (TM) y el texto árabe caligrafiado.

\begin{tabular}{|c|c|}
\hline \multicolumn{2}{|c|}{ Fragmento 1} \\
\hline TO (p. 515) & TM \\
\hline Er ist ein Prophet der Schrift, & Es un profeta de la caligrafía: \\
\hline Gott legte die Kalligraphie in & Alá la puso en su mano \\
\hline seine Hand wie er den Bienen & igual que enseñó a las abejas \\
\hline beibrachte, ihre sechseckigen & a construir sus panales \\
\hline Waben zu bauen. & hexagonales. \\
\hline
\end{tabular}

Las dificultades de traducción en este fragmento estriban en la sinonimia de Schrift y Kalligraphie en alemán, que hace necesaria una omisión en español para evitar la repetición de "caligrafía". Gott se traduce en este contexto como "Alá", ya que el autor de esta cita sobre Ben Muqla es Abu Haiyan al-Tauhidi (enciclopedista y sabio sufí).

\begin{tabular}{|c|c|}
\hline \multicolumn{2}{|c|}{ Fragmento 2} \\
\hline TO (p. 519) & TM \\
\hline $\begin{array}{l}\text { Wähle dir den Weggefährten, } \\
\text { und dann erst den Weg. }\end{array}$ & $\begin{array}{l}\text { Elige primero el compañero y } \\
\text { después el camino. }\end{array}$ \\
\hline بيفرل رتخأ & قيرطلا لبق \\
\hline
\end{tabular}

En la traducción del compuesto alemán Weggefährte (compañero de camino) se ha optado por una elisión parcial para evitar la repetición, al igual que en el fragmento 1. La partícula erst ("solo entonces") se ha compensado semánticamente por el adverbio "primero". En este caso, el 
texto alemán se aproxima mucho más al texto árabe que el español que proponemos. Esto se debe a las posibilidades que ofrece la gramática alemana en comparación con la española, en la que no sería posible ofrecer un texto natural sin el uso del adverbio "primero". La caligrafía correspondiente puede observarse en la figura 1.

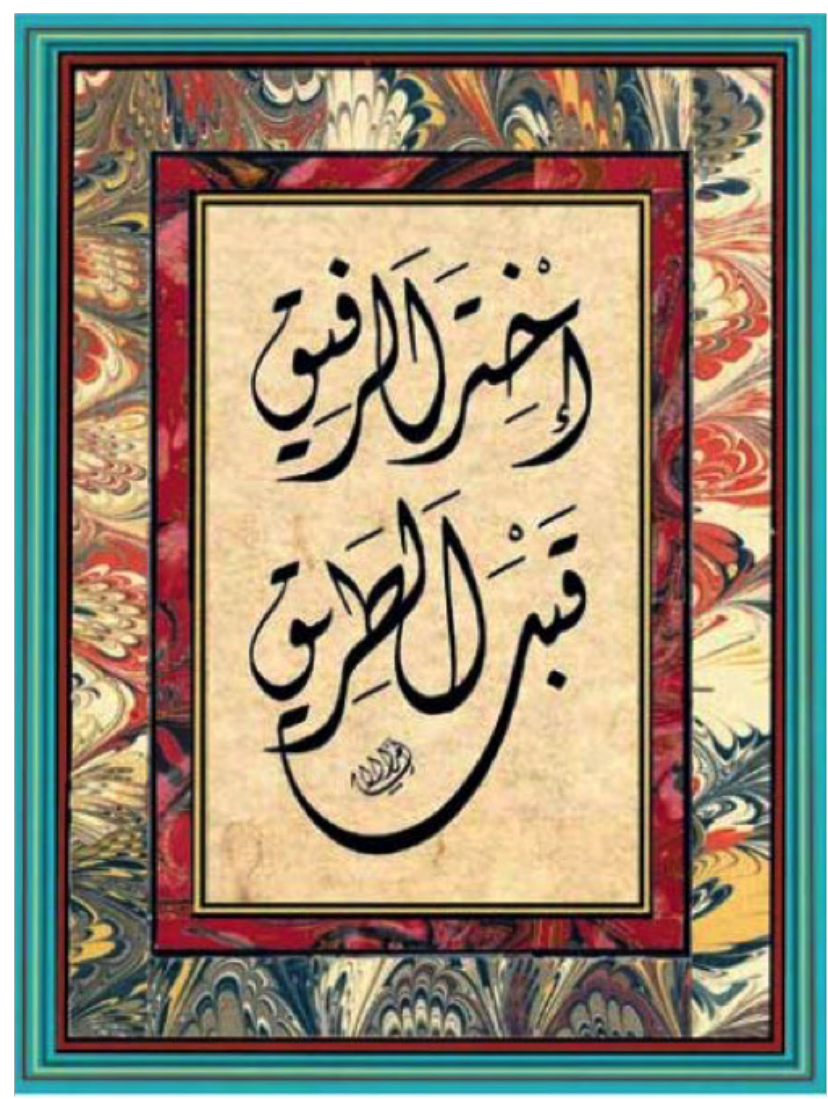

Fig. 1 (Amiralai en Schami, 2012: 519)

\begin{tabular}{|c|c|}
\hline \multicolumn{2}{|c|}{ Fragmento 3} \\
\hline TO (p. 525) & TM \\
\hline Humor und Geduld sind zwei & El humor y la paciencia son \\
\hline Kamele, mit denen du durch & dos camellos con los que \\
\hline jede Wüste kommst. & cruzarás todos los desiertos. \\
\hline \multicolumn{2}{|c|}{ عار حص لك امهب ربعت نالمج حرملاو ربَصلا } \\
\hline
\end{tabular}

En este fragmento, se pluraliza "desiertos" y se emplea el futuro, así como el artículo determinado para "humor" y "paciencia", con el objeto de naturalizar la expresión del TM. Si nos fijamos en el texto original árabe, podemos apreciar que no corresponde al tiempo futuro, ya que en árabe el verbo aparece en presente. En la traducción al español, se hace necesario el uso del tiempo futuro para que la oración se torne natural, ya que el significado del texto original no se pierde y, sin embargo, no podría entenderse bien el matiz propio de esta expresión con el uso del presente. La figura 2 muestra la caligrafía de este fragmento.

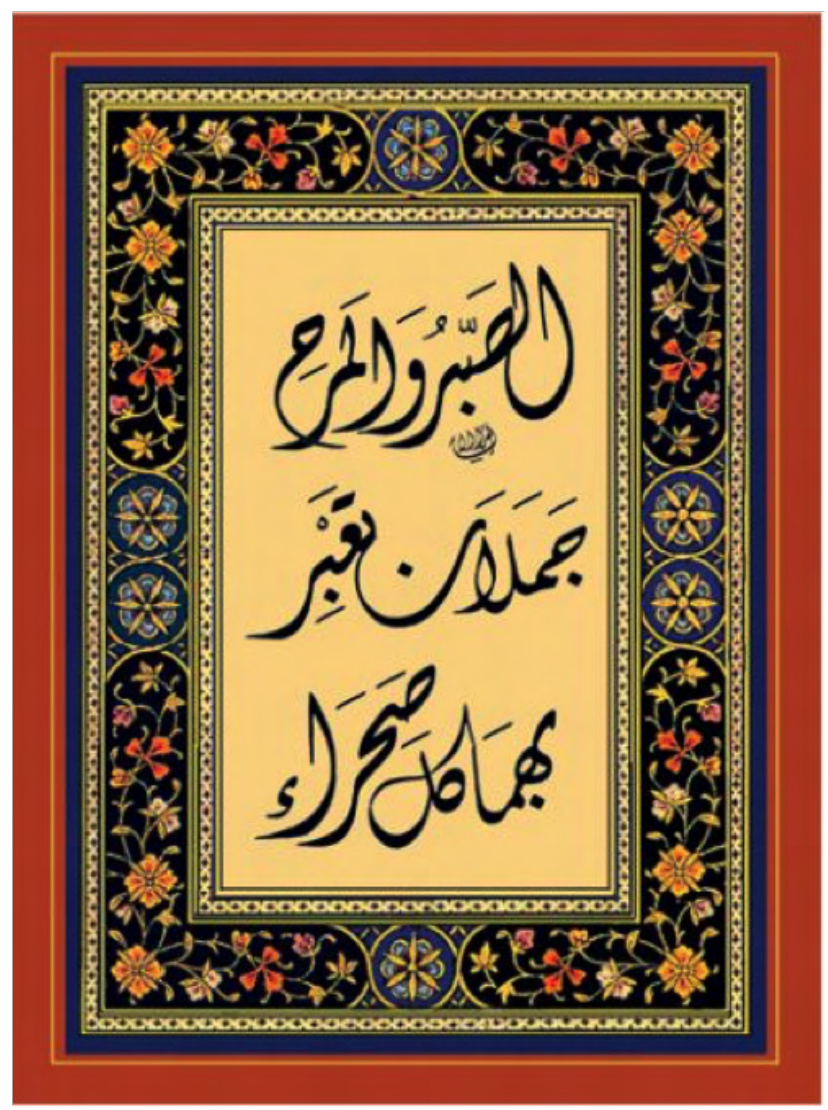

Fig. 2 (Amiralai en Schami, 2012: 525)

\begin{tabular}{|c|c|}
\hline \multicolumn{2}{|c|}{ Fragmento 4} \\
\hline TO (p. 529) & TM \\
\hline $\begin{array}{l}\text { Die Menschen sind des Glückes } \\
\text { Freund } \\
\text { Als es mich für einen halben Tag } \\
\text { verließ, } \\
\text { Suchten Sie ängstlich das Weite. } \\
\text { Oh, Leute kommt zurück, } \\
\text { Das Glück ist mir wieder treu. }\end{array}$ & $\begin{array}{l}\text { Los hombres son amigos de la } \\
\text { suerte. } \\
\text { Cuando esta me dejó apenas } \\
\text { medio día, } \\
\text { se dieron temerosos a la fuga. } \\
\text { Oh, amigos, volved a mí, } \\
\text { la suerte vuelve a serme fiel. }\end{array}$ \\
\hline
\end{tabular}

En este caso, se trata de un poema original del Ben Muqla incluido en el cuerpo del relato para ilustrar la decepción del calígrafo ante su infortunio político. La dificultad del fragmento 4 estriba en la traducción de Menschen y Leute, respectivamente, "hombres" y "amigos". A pesar de que el género está masculinizado en el TM, consideramos que el contexto pragmático de la época justifica su uso, preferible al de vocablos coloquiales o de estilo inadecuado en este contexto como "personas" o "gente". 


\begin{tabular}{|l|l|}
\hline \multicolumn{2}{|c|}{ Fragmento 5 } \\
\hline \multicolumn{1}{|c|}{ TO (p. 531) } & \multicolumn{1}{c|}{ TM } \\
\hline $\begin{array}{l}\text { Ich brachte ihm das Dichten } \\
\text { bei. Sein erster Vers war } \\
\text { gegen mich. }\end{array}$ & Yo le enseñé la poesía. Su \\
\hline \multicolumn{1}{|c|}{ primer verso lo escribió en mi } \\
contra. \\
\hline
\end{tabular}

En este caso, el verbo sustantivado Dichten se ha traducido por el sustantivo "poesía”. Al contrario de lo que sucedía en fragmentos anteriores, el español se aproxima al texto árabe en mayor medida que el alemán. Esto se debe a que en árabe مظن يفاوقل hace referencia a la poesía (literalmente, sistemas de rimas), por lo que su traducción por poesía es más natural que el verbo sustantivado que ofrece el alemán. En la segunda parte de este fragmento, tanto el texto alemán como el español se alejan del árabe.

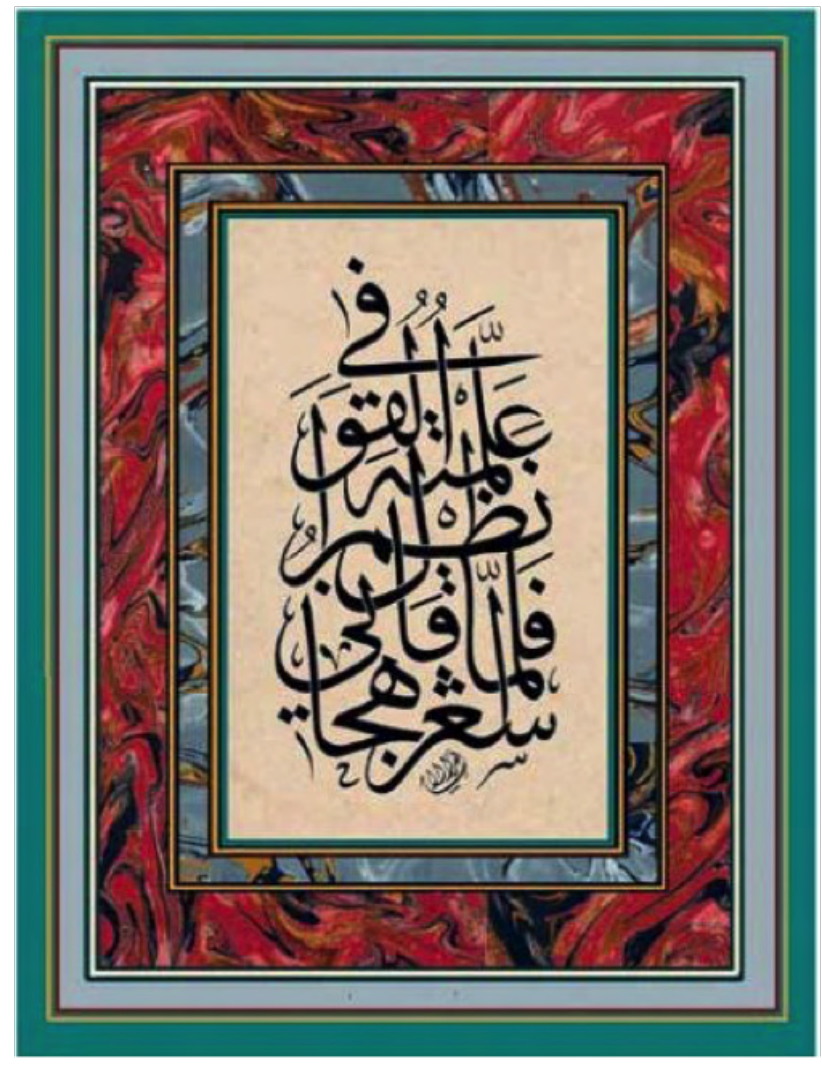

Fig. 3 (Amiralai en Schami, 2012: 531)

En ambos casos, la traducción que permite el árabe por "sátira" ha sido obviada para enfatizar el hecho de que fue empleada contra una persona. Es decir, no se generaliza, sino que se recurre a una traducción alternativa que permite matizar el significado de este fragmento tanto en alemán como en español. Una versión más cercana al árabe sería "Yo le enseñé la rima. Su primer verso fue una sátira hacia mí”.

\begin{tabular}{|c|c|}
\hline \multicolumn{2}{|c|}{ Fragmento 6} \\
\hline TO (p. 535) & TM \\
\hline $\begin{array}{l}\text { Una casa sin libros es un } \\
\text { lugar sin alma. }\end{array}$ & $\begin{array}{l}\text { Yo le enseñé la poesía. Su } \\
\text { primer verso lo escribió en mi } \\
\text { contra. }\end{array}$ \\
\hline
\end{tabular}

En el fragmento 6, variamos el número de Buch a "libros", por considerarlo más natural que la expresión en singular. Sin embargo, en el texto original (Fig. 4) la palabra "libro" (kitāb) aparece en singular, por lo que la traducción alemana resulta más fiel al a TO que la traducción española que proponemos. Esto se debe a que el alemán acepta en esta construcción el uso del singular, mientras que en español este elemento debe aparecer en plural para conservar la naturalidad del TM. Asimismo, hemos optado por la traducción del alemán Seele como alma, en lugar de coger la traducción que ofrece el árabe original (rūḥ) que podría ser también "espíritu".

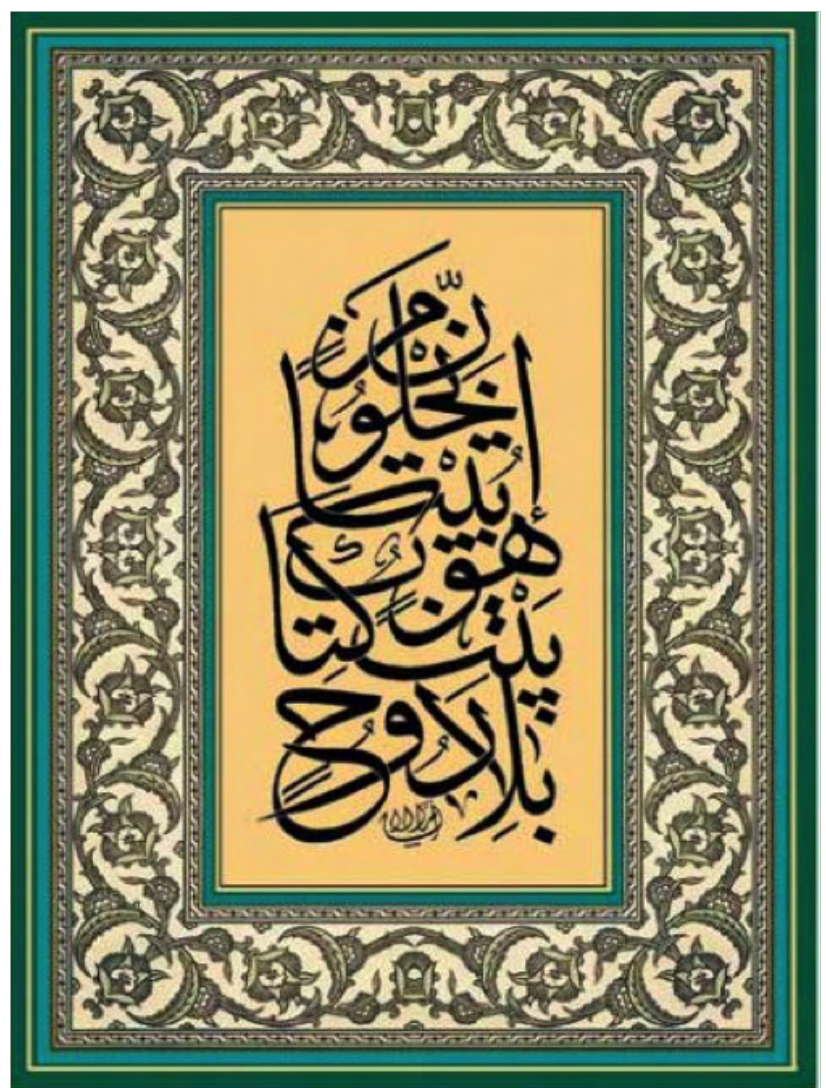

Fig. 4 (Amiralai en Schami, 2012: 535) 


\begin{tabular}{|c|c|}
\hline \multicolumn{2}{|c|}{ Fragmento 7} \\
\hline TO (p. 545) & TM \\
\hline $\begin{array}{l}\text { Wer mit zwei Gesichtern lebt, } \\
\text { stirbt gesichtslos. }\end{array}$ & $\begin{array}{l}\text { Quien vive con dos caras, } \\
\text { muere sin rostro. }\end{array}$ \\
\hline بهجوب شاعن & مل هجو ال تام ر \\
\hline
\end{tabular}

La dificultad en este fragmento (Fig. 7) se produce por el binomio Gesichter - gesichtslos. La derivación en alemán se ha transpuesto mediante el uso de sinónimos en español. En ambos casos, el recurso para realizar la traducción del texto árabe ha sido el adecuado. El fragmento en árabe ofrece, por un lado, el sustantivo "cara" وج que aparece en forma dual y, por otro, el pronombre singular en tercera persona. Esta ausencia del sustantivo en la segunda parte del fragmento árabe ha tenido que resolverse tanto en alemán como en español con otro elemento (gesichtslos y "sin rostro", respectivamente) para que no se pierda información contenida en el texto árabe y se transmita a ambas lenguas el significado de este fragmento.

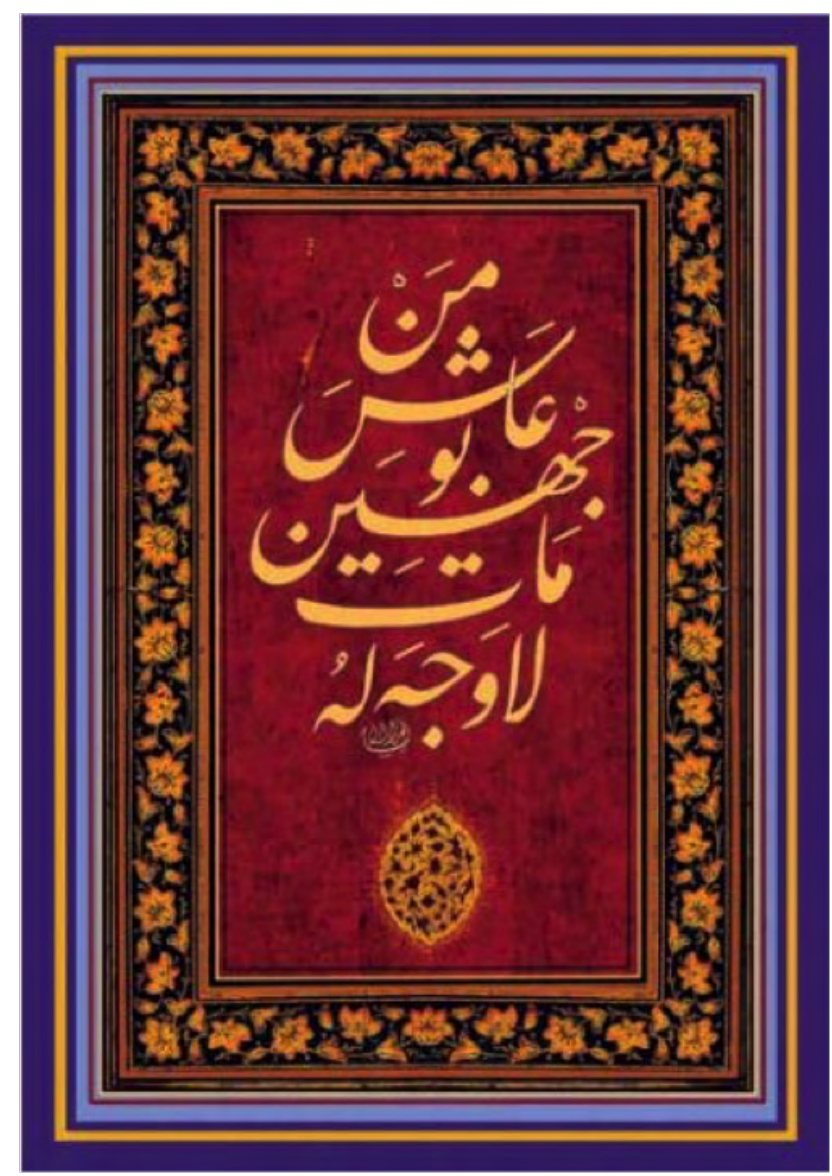

Fig. 5 (Amiralai en Schami, 2012: 545)

\subsection{El lenguaje técnico: la caligrafía}

El primer epígrafe del epílogo, incluido también en el cuerpo de la novela, contiene datos biográficos sobre Ben Muqla y algunas explicaciones preliminares sobre la reforma ortográfica que este llevó a cabo:

El alif, la $a$ árabe, es un trazo vertical, y Ben Muqla lo eligió como referencia de las demás letras. Desde entonces, todos los calígrafos fijan al principio la longitud del alif para la escritura elegida. El cálculo se hace con puntos verticales sucesivos. El punto se rige a su vez por la pluma empleada y se realiza apretando la pluma contra el papel. Todas las demás letras, ya sean horizontales o verticales, adoptan un tamaño que Ben Muqla calculó y fijó con un número de puntos. También los redondeos de algunas letras se sitúan en un círculo cuyo diámetro corresponde a la longitud del alif. La observancia de esa medida corresponde a la observancia del ritmo en una obra musical. Solo a través de ella la escritura resulta armoniosa y se convierte en música para el ojo. Después de años de práctica, los maestros dominan esas reglas de manera automática. Pero los puntos permiten comprobar rápidamente si la proporción es buena. (Schami 2012: 235).

Los sucesivos epígrafes describen en detalle diversos aspectos de la caligrafía árabe, comenzando por sus orígenes a partir del arameo y del alfabeto nabateo (Schami 2012: 532).

\begin{tabular}{|l|l|}
\hline \multicolumn{2}{|c|}{ Fragmento 8 } \\
\hline \multicolumn{1}{|c|}{ TO (p. 533) } & \multicolumn{1}{|c|}{ TM } \\
\hline Arabisch wird von rechts & El árabe se escribe de derecha \\
nach links geschrieben. Das & a izquierda. El alfabeto \\
Alphabet hat 25 Konsonanten & tiene 25 consonantes y tres \\
und drei lange Vokale A, & vocales largas: a, i, u. Las \\
I und U. Die Kurzvokale & vocales cortas, en los escritos \\
wurden in den alten & antiguos, se escribían encima \\
Schriften über und unter & y debajo de las letras, lo que \\
den Buchstaben gezeichnet, & posibilitaba una correcta \\
was ein fehlerfreies Lesen & lectura. En la escritura \\
ermöglichte. In der modernen & moderna se prescinde de \\
Schrift verzichtet man auf & estos detalles. \\
diese Nuancen. & \\
\hline
\end{tabular}


Este fragmento no ofrece gran dificultad en la traducción. La información que aparece en TO se ha conservado en TM, por lo que se ha descrito la composición del alfabeto en árabe, conocido como alifato, así como el uso de las vocales largas y cortas que resultan imprescindibles en la escritura árabe. Es cierto, como Schami indica, que el uso de las vocales cortas -y nos atrevemos a incluir aquí otros elementos de la escritura árabe como el sukūn, entre otros- ha ido desapareciendo en la escritura moderna hasta el punto de que no se utilizan nada más que en aquellos casos en los que su ausencia pueda llevar a una lectura o comprensión errónea de una palabra. En este caso, nos estamos refiriendo, y así creemos que lo hace Schami, al denominado árabe clásico o fușha.

\begin{tabular}{|c|c|}
\hline \multicolumn{2}{|c|}{ Fragmento 9} \\
\hline TO (p. 533) & TM \\
\hline Die einzelnen Buchstaben & En la caligrafía árabe, las \\
\hline werden in der arabischen & distintas letras siempre se \\
\hline Schrift immer miteinander & unen, tanto en la escritura \\
\hline verbunden, sowohl beim & manual como en la impresa. \\
\hline handschriftlichen Schreiben & Cada letra tiene cuatro \\
\hline als auch im Druck. Jeder & formas, según si va al \\
\hline Buchstabe hat vier Formen, je & inicio, en medio, al final o \\
\hline nachdem, ob er am Anfang, & aislada. El alfabeto latino, \\
\hline in der Mitte, am Ende oder & por el contrario, solo tiene \\
\hline frei steht. Die lateinische & dos formas: mayúscula y \\
\hline Schrift kennt dagegen nur & minúscula. La dificultad es \\
\hline zwei Formen, Majuskel und & que 22 letras árabes pueden \\
\hline Minuskel. Erschwerend & unirse desde ambos lados, \\
\hline kommt hinzu, dass sich 22 & mientras que seis (و ذدز ر أ) \\
\hline Buchstaben im Arabischen & no pueden unirse hacia la \\
\hline von beiden Seiten verbinden & izquierda, por lo que si se \\
\hline lassen, sechs Buchstaben & encuentran en mitad de una \\
\hline (و ذد ز ز أ) jedoch nicht & palabra se escribe la letra a su \\
\hline nach links. Wenn sie also & izquierda como si estuviera \\
\hline in der Mitte eines Wortes & al principio de la palabra. $\mathrm{Al}$ \\
\hline vorkommen, wird der & contrario que en el alfabeto \\
\hline Buchstabe links von ihnen & latino, el ligado ${ }^{1}$ (unión entre \\
\hline so geschrieben, als stünde er & las letras) tiene un papel \\
\hline am Anfang eines Wortes. Im & esencial en el diseño de la \\
\hline Gegensatz zur lateinischen & caligrafía árabe. \\
\hline Schrift spielen Ligaturen & \\
\hline (Verbindung zwischen & $\begin{array}{l}{ }^{1} \text { Ver http://www.cervantesvirtual. } \\
\text { com/obra-visor/arte-de-la-escritura- }\end{array}$ \\
\hline den Buchstaben) bei der & $\begin{array}{l}\text { y-de-la-caligrafia-teoria-y-practica--0/ } \\
\text { html/ff0f1954-82b1-11df-acc7- }\end{array}$ \\
\hline Gestaltung der arabischen & 002185ce6064_24.html (Consulta: \\
\hline Schrift eine wesentliche Rolle. & \\
\hline
\end{tabular}

Este fragmento ofrece información sobre la escritura árabe. En este caso, Schami está haciendo referencia al hecho de que las letras árabes se unen entre ellas y ofrecen una caligrafía diferente en función del lugar que ocupen en la palabra. Además, resalta el autor, existen varias letras que no pueden unirse a la siguiente por la izquierda, lo que condiciona la escritura de la letra que aparece a continuación. Este trazo enlazado que ofrecen las letras árabes propicia, sin duda, que la caligrafía pueda someterse a un trazo más cuidado y, por tanto, más elaborado que en el caso de las letras de alfabetos latinos en los que las letras no van unidas y la caligrafía se permite jugar únicamente con la alternancia de la mayúscula y la minúscula.

En el tercer apartado del epílogo, se describen los seis estilos clásicos de la caligrafía árabe desarrollados a lo largo de los siglos:

\begin{tabular}{|c|c|}
\hline \multicolumn{2}{|c|}{ Fragmento 10} \\
\hline TO (p. 536) & TM \\
\hline $\begin{array}{l}\text { Kufi: so genannt nach } \\
\text { der irakischen Stadt al } \\
\text { Kufa. Sie erreichte ihre } \\
\text { Vollkommenheit bereits in } \\
\text { der zweiten Hälfte des } 8 . \\
\text { Jahrhunderts. Dieser Stil hat } \\
\text { ein eckiges Schriftbild, das } \\
\text { sakral wirkt und Minarette } \\
\text { anzudeuten scheint. Er ist } \\
\text { sehr beliebt in der Bauweise } \\
\text { sowohl von Moscheen als } \\
\text { auch von Palästen, aber } \\
\text { auch bei der Gestaltung von } \\
\text { Alltagsgegenständen. Eine } \\
\text { beliebte Variante dieses Stils } \\
\text { ist der florale Kufi-Stil. Hier } \\
\text { ragen Pflanzen und Blumen } \\
\text { aus den Buchstaben und } \\
\text { ergeben eine ausgeprägte } \\
\text { Ornamentik, aber sie } \\
\text { erschweren das Lesen. Bei } \\
\text { einer weiteren Variante, dem } \\
\text { geflochtenen Kufi, können } \\
\text { nur noch Experten das } \\
\text { Ornament entziffern. }\end{array}$ & $\begin{array}{l}\text { Küfï: llamado así por la } \\
\text { ciudad iraquí de Kufa. } \\
\text { Alcanzó su esplendor ya en } \\
\text { la segunda mitad del s. VIII. } \\
\text { Este estilo tiene un aspecto } \\
\text { anguloso y sacro que parece } \\
\text { imitar los minaretes. Es muy } \\
\text { popular en la construcción } \\
\text { tanto de mezquitas como } \\
\text { de palacios, así como en } \\
\text { los objetos cotidianos. Una } \\
\text { variante de este estilo es el } \\
\text { cúfico floreciente, en el que } \\
\text { surgen plantas y flores de las } \\
\text { letras con marcado carácter } \\
\text { ornamental, pero difícil de } \\
\text { leer. Otra variante, el cúfico } \\
\text { cuadrado, solo puede ser } \\
\text { descifrada por los expertos. }\end{array}$ \\
\hline
\end{tabular}


En la traducción de este fragmento no se han encontrado elementos que hayan revestido dificultad. Sin embargo, hemos optado por la transcripción fonética del nombre de este estilo caligráfico $(K \bar{u} f i ̂)$, siguiendo las normas desarrolladas para ello, porque nos resulta más adecuado el uso del nombre transcrito. En las ocasiones siguientes que aparece el nombre de este estilo caligráfico, hemos optado por el uso de su nombre en español "cúfico", como sucede en el TO en alemán.

\begin{tabular}{|c|c|}
\hline \multicolumn{2}{|c|}{ Fragmento11 } \\
\hline TO (p. 536) & TM \\
\hline Thuluth, (the wie im & Thuluth (pronunciado como \\
\hline Englischen; auch Tulut & la th inglesa, también llamado \\
\hline oder-im Türkischen-Sülüs & tulut o sülüs en turco): el \\
\hline genannt): Der Name bedeutet & nombre significa "un tercio" \\
\hline ein Drittel und bezog sich & y se refería antiguamente al \\
\hline früher auf die Breite der & grosor de la punta del cálamo \\
\hline Rohrfederspitze, mit der & con el que se escribía. El \\
\hline geschrieben wurde. Die & mayor tenía 24 trazos finos de \\
\hline breiteste war 24 Haar breit, & grosor, en caligrafía thuluth \\
\hline die Thuluth-Schrift nur acht. & solo ocho. \\
\hline
\end{tabular}

En este fragmento se describe el sistema de caligrafía thuluth. Durante la traducción del mismo hemos encontrado que la traducción del sustantivo alemán Haar no podía hacerse al español por "pelo", confusión posible si no se tiene un conocimiento previo del campo de la caligrafía. Este sustantivo hace referencia al grosor que tendría el cálamo con el que se ejecutaba el trazo caligráfico en cuestión, y que en este caso se mide en Haarstriche o "trazos finos" (Ernst, 2006: 581). De hecho, es en este elemento donde reside la característica principal de este sistema caligráfico, ya que se diferencia de la caligrafía tumar por emplearse un tercio del grosor de su trazo.

\begin{tabular}{|l|l|}
\hline \multicolumn{2}{|c|}{ Fragmento12 } \\
\hline \multicolumn{1}{|c|}{ TO (p. 536) } & \multicolumn{1}{|c|}{ TM } \\
\hline Nas-chi (auch Naß-ch, Neskh, & Nasjī (también naskh, nasj): \\
Neskhi): Vom Meister aller & creada por el maestro de los \\
Meister Ibn Muqla im 10. & maestros, Ben Muqla, en el \\
Jahrhundert kreiert, um die & s. X, para facilitar el trabajo \\
Arbeit der Kopisten (arab. & de los copistas (en árabe, nasj \\
nasacha = kopieren) zu & significa copiar) y, sobre todo, \\
erleichtern und vor allem & la lectura clara. Hoy día casi \\
das eindeutige Lesen zu & todos los libros se escriben en \\
ermöglichen. Heute werden & este estilo, elegante y claro. \\
fast alle Bücher in diesem Stil & \\
geschrieben. Elegant und klar. & \\
\hline
\end{tabular}

La escritura naskhī o nasjī, en su transcripción fonética, es sin duda el sistema caligráfico más utilizado desde que Ben Muqla lo creó. Esto se debe al trazo claro de las letras que permite que la lectura se lleve a cabo sin ninguna dificultad. Como se ha adelantado con anterioridad, este sistema fue empleado durante el s. XI para escribir el Corán, popularizándose así el uso de la caligrafía naskhī y permitiendo la lectura del Corán sin dificultades mayores. En cuanto a la traducción de este fragmento, no hemos encontrado ningún elemento destacable. En cualquier caso, hemos optado por la transcripción fonética del nombre de este sistema caligráfico, haciendo uso para ello de dos de las formas posibles que ofrece esta palabra, según el sistema de transcripción que se esté empleando. Esta doble transcripción ha sido posible gracias al TO en el que Schami ofrece cuatro formas diferentes de transcripción para este mismo término (Nas-chi, Naß-ch, Neskh, Neskhi), siguiendo para ello las normas fonéticas en alemán. En nuestra traducción hemos decidido suprimir una de ellas ( $\mathrm{Na \beta}$ - $\mathrm{ch}$ ) porque entendemos que se encuentra presente en TO para que el lector alemán pueda conocer la pronunciación de este término sin tener conocimientos previos de fonética. Sin embargo, en español esta transcripción es innecesaria y, mucho más, la transcripción alemana.

\begin{tabular}{|l|l|}
\hline \multicolumn{2}{|c|}{ Fragmento13 } \\
\hline \multicolumn{1}{|c|}{ TO (p. 538) } & \multicolumn{1}{|c|}{ TM } \\
\hline Riq’a (auch Ruq'a, Riqaa & Riqa’i (también ruq’a o riq’a): \\
und Riqa'i): Wurde von den & desarrollado por los calígrafos \\
osmanischen Kalligraphen & otomanos, que querían la \\
entwickelt, die die & mayor simplificación posible. \\
größtmögliche Vereinfachung & Es una caligrafía pequeña, \\
erreichen wollten. Die & comprimida y sin adornos. Se \\
Schrift ist klein, gedrängt, & adecúa a la escritura manual \\
schmucklos. Sie ist für das & y se extendió con rapidez. \\
handschriftliche Schreiben & A menudo se escriben los \\
geeignet und verbreitete sich & titulares de los periódicos con \\
schnell. Häufig werden die & este conocido estilo. \\
Schlagzeilen einer Zeitung & \\
mit dieser vertrauten Schrift & \\
geschrieben. & \\
\hline
\end{tabular}

$\mathrm{Al}$ igual que en el fragmento anterior, no hemos encontrado una mayor dificultad en el desarrollo de la traducción, mas se ha tenido que determinar la transcripción fonética del nombre de este estilo de caligrafía atendiendo para ello a la fonética española. 


\begin{tabular}{|l|l|}
\hline \multicolumn{2}{|c|}{ Fragmento 14 } \\
\hline \multicolumn{1}{|c|}{ TO (p. 538) } & \multicolumn{1}{|c|}{ TM } \\
\hline Farsi (auch Ta'liq und & Fārsī (también Ta’līq o \\
Nasta'liq genannt): Luftige & Nasta’līq): caligrafía ligera y \\
elegante Schrift. Der Strich & elegante. El trazo es delgado y \\
ist schlank und dynamisch, & dinámico, a menudo oblicuo. \\
oft schräg. Die wenigen & Los ángulos son escasos y \\
Spitzen sind oft gerundet. & a menudo redondeados. La \\
Großzügige, breite Dehnung & extensión generosa y amplia \\
der horizontalen Buchstaben, & de las letras horizontales \\
was der visuellen Musik der & confiere un tono tranquilo \\
Schrift im Rhythmus der & al ritmo de las letras en la \\
Buchstaben einen ruhigen & música visual de la caligrafía. \\
Klang verleiht. Heute & Hoy día es el estilo de \\
beherrscht dieser Stil das & caligrafía dominante en Irán. \\
Schriftbild in Iran. & \\
\hline
\end{tabular}

En este caso se describe el estilo de caligrafía conocido comofārsī. Hemos optado nuevamente por seguir las normas de transcripción fonética para los diferentes nombres con los que puede conocerse este estilo caligráfico. En cuanto a la traducción, los elementos propios de la caligrafía que aparecen no han supuesto una dificultad importante a la hora de traducirlos.

\begin{tabular}{|l|l|}
\hline \multicolumn{2}{|c|}{ Fragmento 15 } \\
\hline \multicolumn{1}{|c|}{ TO (p. 538) } & \multicolumn{1}{c|}{ TM } \\
\hline Diwani: Wurde in den & Dīwānì: se empleaba en la \\
osmanischen Staatskanzleien & administración (dīwān) \\
(Diwan) verwendet. Diwani & otomana. El dīwānī es una \\
ist eine majestätische Schrift, & caligrafía majestuosa, cuyas \\
deren Buchstaben oft & letras a menudo siguen un \\
einem Kreis folgen, dessen & círculo cuyo diámetro viene \\
Durchmesser die Höhe des & dado por la longitud del alif \\
Alif bestimmt (siehe S. 518). & (ver pág. 518). \\
\hline
\end{tabular}

La caligrafía dīwānì, descrita en este fragmento no ha sido dificultosa a la hora de traducirlo. Nuevamente, y como ha sucedido en otros casos, hemos transcrito fonéticamente el nombre de esta caligrafía.

\begin{tabular}{|l|l|}
\hline \multicolumn{2}{|c|}{ Fragmento 16 } \\
\hline \multicolumn{1}{|c|}{ TO (p. 538) } & \multicolumn{1}{|c|}{ TM } \\
\hline Erghrha:Stil ist ausgestorben. & Tughra: este estilo está en \\
Fingerabdruck und war dem & desuso. Se parecía a una \\
huella dactilar y estaba \\
Sultan vorbehaltet. In der & reservado al sultán. En su \\
und mestaltung waren Name & realización se integraban el \\
majestätische Beiname des & nombre y a veces también \\
Sultans integriert. Später & el sobrenombre real del \\
wurden auch religiöse & sultán. Más tarde también se \\
Sprüche in diesem Stil & escribieron citas religiosas en \\
geschrieben und so zu & este estilo y se convirtió en un \\
einer Ikone erhoben. Der & icono. El trazo no seguía la \\
Schriftzug folgte nicht der & regla de derecha a izquierda, \\
Regel des Schreibens von & sino que transcurría como lo \\
rechts nach links, sondern & exigía la figura.inante en Irán. \\
verlief, wie es die Figur & \\
verlangte. & \\
\hline
\end{tabular}

En este fragmento se describe el sistema de caligrafía denominado tughra. La traducción del fragmento no presenta elementos destacables.

\begin{tabular}{|c|c|}
\hline \multicolumn{2}{|c|}{ Fragmento 17} \\
\hline TO (p. 540) & TM \\
\hline Da sie immer gebunden & Dado que las letras siempre \\
\hline geschrieben wird, spielt die & se enlazan, la longitud del \\
\hline Länge der Bindung zwischen & enlace entre ellas (ligado) \\
\hline den Buchstaben (Ligatur) & tiene un papel especial en la \\
\hline bei der Komposition eine & composición. La extensión \\
\hline besondere Rolle. Die & y acortamiento de este \\
\hline Dehnung und Kürzung & enlace es al ojo lo que la \\
\hline dieser Bindung ist für das & mayor o menor duración \\
\hline Auge wie die Verlängerung & de un sonido al oído. El alif, \\
\hline oder Kürzung eines Tones & un trazo vertical en árabe, \\
\hline für das Ohr. Das A(lif), im & se transforma en la barra \\
\hline Arabischen ein senkrechter & de compás que marca el \\
\hline Strich, verwandelt sich in & ritmo de la música. Y, dado \\
\hline einen Taktstrich für den & que la altura de la letra \\
\hline Rhythmus der Musik. Aber & alif, según las normas de la \\
\hline da wiederum die Größe des & proporcióndetermina la de las \\
\hline Buchstaben A(lif) der ... & demás letras, participa ... \\
\hline
\end{tabular}




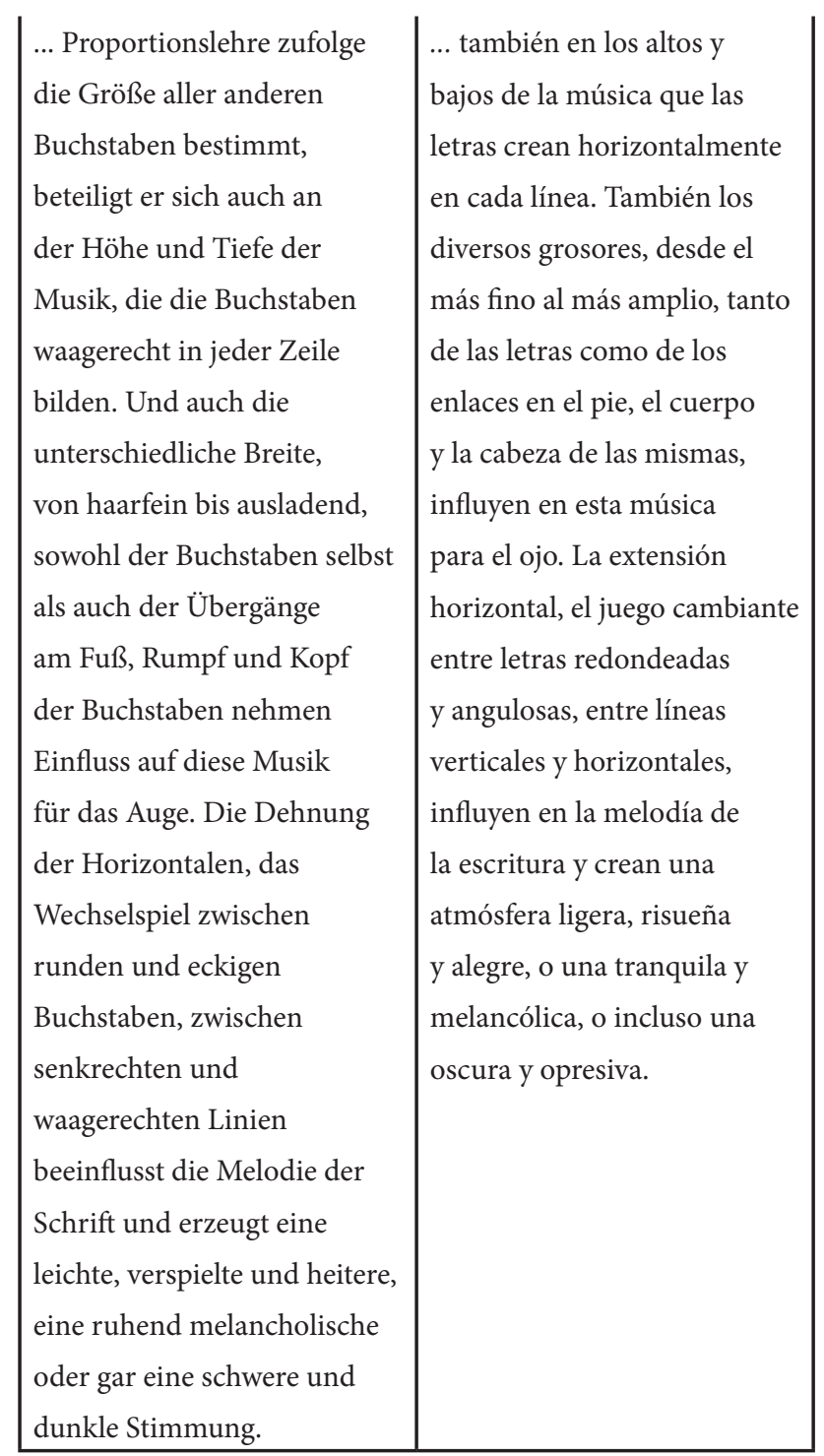

En este fragmento, la mayor dificultad estriba en la combinación de las descripciones caligráficas técnicas con el lenguaje literario. Además de los tecnicismos "ligado", "enlace", "extensión" o "acortamiento", encontramos un paralelismo de la caligrafía con el lenguaje musical. Este incide en la atmósfera y el estado de ánimo, descritos con palabras como verspielt, que preferimos no traducir por su sentido literal (“juguetona”), dado que resta comportaría una variación diafásica más coloquial. En su lugar, hemos optado por "risueña" para conservar tanto el sentido como la variedad lingüística.

\begin{tabular}{|l|l|}
\hline \multicolumn{2}{|c|}{ Fragmento18 } \\
\hline \multicolumn{1}{|c|}{ TO (p. 540) } & \multicolumn{1}{|c|}{ TM } \\
\hline $\begin{array}{l}\text { Und, möchte man sorgfältig } \\
\text { mit den Buchstaben } \\
\text { musizieren, so erfordert }\end{array}$ & Y, si se quiere crear una \\
música cuidada con las letras, \\
los espacios entre las
\end{tabular}

\begin{tabular}{|l|l|}
.... die Leere zwischen den & ... letras y palabras requieren \\
Buchstaben und Worten ein & una habilidad aún mayor. \\
Die leeren Räßeres Geschick. & Los vacíos de la caligrafía \\
Kalligraphie sind Augenblicke & son momentos de silencio. \\
der Stille. Und wie die & también la caligrafía recurre a \\
arabische Musik setzt auch & la repetición de determinados \\
die Kalligraphie auf die & elementos, que no solo \\
Wiederholung bestimmter & requieren un baile del cuerpo \\
Elemente, die nicht nur den & y el alma, sino también \\
Tanz von Körper und Seele, & desprenderse de lo terrenal y \\
sondern auch das Loslösen & alcanzar otras esferas. \\
vom Irdischen und das & \\
Erreichen anderer Sphären & \\
fördert. & \\
\hline
\end{tabular}

Schami considera que esta "música para los ojos" no requiere conocimientos del idioma para ser disfrutada: "Und so wie das Ohr die Musik genießt, genießt das Auge die arabische Kalligraphie, ohne sie zu verstehen". (Schami 2012: 540). Como ejemplo indica la figura de Goethe, que sin haber estudiado el árabe realizaba ejercicios de escritura en este idioma:

\begin{tabular}{|c|c|}
\hline \multicolumn{2}{|c|}{ Fragmento 19} \\
\hline TO (p. 540-541) & TM \\
\hline $\begin{array}{l}\text { Der geniale Goethe hat sich, } \\
\text { wie sein Nachlass zeigt, } \\
\text { darin geübt, Arabisch zu } \\
\text { schreiben, ohne dass er je } \\
\text { Arabisch hätte lesen können. } \\
\text { Vor allem in der Zeit, als er } \\
\text { sich intensiv mit dem Orient } \\
\text { und dem „Westöstlichen } \\
\text { Divan“ beschäftigte. Warum? } \\
\text { Goethe hat erkannt, dass das } \\
\text { Arabische in seiner Form } \\
\text { wie keine andere Schrift das } \\
\text { Wesen der arabischen Kultur } \\
\text { vermittelt, auch wenn der } \\
\text { Inhalt dem Nichtkundigen } \\
\text { verborgen bleibt. }\end{array}$ & $\begin{array}{l}\text { El genial Goethe se ejercitaba, } \\
\text { como muestran sus obras } \\
\text { póstumas, escribiendo en } \\
\text { árabe, sin haber aprendido } \\
\text { nunca a leer este idioma. } \\
\text { Practicó la caligrafía } \\
\text { especialmente durante la } \\
\text { época en la que se interesó } \\
\text { por Oriente y trabajó en } \\
\text { su poemario El Diván de } \\
\text { Oriente y Occidente. ¿Por } \\
\text { qué? Goethe se dio cuenta } \\
\text { de que el árabe, en su forma } \\
\text { escrita, transmite la esencia } \\
\text { de la cultura árabe como no } \\
\text { puede hacerlo ningún otro } \\
\text { alfabeto, incluso aunque el } \\
\text { significado de la escritura } \\
\text { permanezca oculto para los } \\
\text { no iniciados. }\end{array}$ \\
\hline
\end{tabular}


En el siguiente fragmento se comenta el número impreciso de caracteres árabes:

\begin{tabular}{|c|c|}
\hline \multicolumn{2}{|c|}{ Fragmento 20} \\
\hline TO (p. 542) & TM \\
\hline Die arabische Sprache & La lengua árabe tiene \\
\hline verfügt über genau & exactamente veintiocho \\
\hline achtundzwanzig Buchstaben. & letras. La supuesta letra \\
\hline Der vermeintliche & número veintinueve es $L A$ y \\
\hline neunundzwanzigste & se compone -al igual que su \\
\hline Buchstabe ist $L A$ und besteht & transcripción en el alfabeto \\
\hline -wie seine Wiedergabe in & latino- de las dos letras $l$ y $a$. \\
\hline lateinischen Buchstaben- aus & Por tanto, no se trata de una \\
\hline zwei Buchstaben $L$ und $A$. & letra, sino de una palabra que \\
\hline Also ist das kein Buchstabe, & significa "no". \\
\hline sondern ein Wort und & \\
\hline bedeutet „nein“ oder „nicht“. & \\
\hline
\end{tabular}

En este fragmento, el elemento más destacable ha sido la supresión en TM de una de las palabras alemanas para referirse a "no" puesto que en español solo existe una forma única para la negación. Del mismo modo, desde el punto de vista ortotipográfico, las letras no se mayusculizan en español y se emplean las comillas latinas.

\begin{tabular}{|c|c|}
\hline \multicolumn{2}{|c|}{ Fragmento 21} \\
\hline TO (p. 544-545) & TM \\
\hline Der Koran ist für über & El Corán es el libro sagrado \\
\hline eine Milliarde Menschen & de más de mil millones de \\
\hline das heilige Buch und soll & personas y debe mantenerse \\
\hline unangetastet bleiben, aber & inalterado, pero la lengua \\
\hline die arabische Sprache des & árabe cotidiana, del \\
\hline Alltags, des Wissens, der & conocimiento, de la poesía, \\
\hline Poesie bedarf radikaler & requiere reformas radicales. \\
\hline Reformen. Als Erstes muss & Lo primero es reformar los \\
\hline man die Bausteine der & bloques de la lengua: las \\
\hline Sprache, die Buchstaben, & letras. A la caligrafía le faltan \\
\hline reformieren. Der Schrift & varias letras, como e, o, p y \\
\hline fehlen ein paar Buchstaben, & $\mathrm{w}$, para poder comunicarse \\
\hline wie $\mathrm{E}, \mathrm{O}, \mathrm{P}$ und $\mathrm{W}$, um mit & con facilidad con las \\
\hline den anderen Sprachen leicht & demás lenguas y asumir \\
\hline zu kommunizieren, ihre & sus expresiones sin tener \\
\hline Ausdrücke aufzunehmen, & que escribir las palabras en \\
\hline ohne das Wort in Latein & caracteres latinos. \\
\hline schreiben zu müssen. & \\
\hline
\end{tabular}

En este segmento, la traducción ha resultado sencilla. Sin embargo, la alusión a las letras p y w que realiza Schami puede no entenderse bien en el TM, puesto que son letras que se hacen imprescindibles en la lengua alemana pero que no son de uso tan frecuente en español como lo son en esta.

En las siguientes páginas, Schami describe las posibles opciones para solucionar el problema de los neologismos en árabe: desde los préstamos del francés y el inglés, hasta la renuncia a escribir en árabe clásico y primar los dialectos, o el recurso al alfabeto latino, lo que supondría renunciar a una parte importante de la cultura árabe: "Aber dieser Vorschlag ist für die Araber unannehmbar, weil die arabischen Buchstaben eine kardinale Stütze der ganzen arabischen Kultur sind." (Schami 2012: 547).

Del mismo modo, el autor describe la reforma propugnada por Sa’id al-Sakkar en Bagdad durante la dictadura de Sadam Hussein:

\begin{tabular}{|c|c|}
\hline \multicolumn{2}{|c|}{ Fragmento 22} \\
\hline TO (p. 548) & TM \\
\hline Der irakische Dichter, Maler & El poeta, pintor y calígrafo \\
\hline und Kalligraph Muhammad & iraquí Mohammed Sa’īd \\
\hline Said al Sakkar entwickelte & al-Sakkar desarrolló tras \\
\hline nach jahrlanger, mühseliger & años de esforzado trabajo \\
\hline Arbeit ein, wie er es nannte, & un "alfabeto concentrado", \\
\hline „konzentriertes Alphabet“ & según sus palabras, con tan \\
\hline mit nur fünfzehn Buchstaben. & solo quince letras. Cada \\
\hline Jeder Buchstabe hat eine & letra tiene una única forma, \\
\hline einzige Form, gleich wo er & independientemente del lugar \\
\hline im Wort vorkommt. Sakkar & que ocupa en la palabra. \\
\hline nannte es „Fesseln des & Sakkar lo llamó "romper \\
\hline Platzes brechen“. Dies sollte & las ataduras de la posición". \\
\hline das Lesen und Erlernen der & Con ello pretendía facilitar \\
\hline Buchstaben und Worte und & la lectura y el aprendizaje de \\
\hline das Computerschreiben sowie & las letras y palabras, así como \\
\hline die Druckarbeit erleichtern. & la escritura a ordenador y la \\
\hline & impresión. \\
\hline
\end{tabular}

Al-Sakkar tuvo que exiliarse en 1978 a París por esta reforma radical del alfabeto, que, sin embargo, tampoco cuenta con la aprobación de Schami, quien aboga por ampliarlo e incluir los caracteres adicionales necesarios:

\begin{tabular}{|c|c|}
\hline \multicolumn{2}{|c|}{ Fragmento 23 } \\
\hline TO (p. 540) & TM \\
\hline Doch bei allem Respekt vor & Con todos los respetos hacia \\
\hline
\end{tabular}




\begin{tabular}{|l|l|}
... dieser großen Bemühung & ... este ímprobo esfuerzo, \\
muss gesagt werden, dass die & debo decir que reducir las \\
Reduzierung der Buchstaben & letras empobrece una lengua \\
eine Sprache verarmt und & y daña su elegancia. También \\
ihre Eleganz schädigt. Das & causa más problemas de gran \\
wirft auch weitere große & calado. Habría que transcribir \\
Probleme auf. Man müsste & nuevamente el Corán y \\
den Koran neu kopieren und & enseñar estas modificaciones \\
diese Veränderungen der & al mundo islámico, de lo \\
islamischen Welt beibringen, \\
sonst entsteht das größte & contrario se produce el mayor \\
Chaos aller Zeiten: zwei oder & o tres alfabetos que coexisten \\
drei Alphabete existieren & y compiten y solo causan \\
neben- und gegeneinander & confusión. \\
und stiften nur Verwirrung. & No, la ampliación del alfabeto \\
Nein, die Erweiterung & es la solución razonable. El \\
des Alphabets ist die & Corán puede mantenerse \\
vernünftige Lösung. Der & inalterado, y la lengua \\
Koran kann unangetastet & cotidiana, de la poesía, de la \\
bleiben, und das Alphabet \\
des Alltags, der Poesie, der \\
Wissenschaft erweitert sich \\
um vier Buchstaben. Perser, \\
Pakistanis, Afghanen und \\
andere islamische Völker \\
haben das Alphabet längst \\
erweitert, ohne weniger gute \\
Muslime geworden zu sein. \\
paquistaníes, los afganos y \\
otros pueblos islámicos hace \\
mucho que ampliaron sus \\
alfabetos, y no por ello son \\
peores musulmanes.
\end{tabular}

A continuación, ofrecemos un glosario de palabras técnicas relacionadas con el campo de la caligrafía árabe que aparecen en el fragmento analizado en nuestro trabajo:

\begin{tabular}{|c|c|}
\hline ALEMÁN & ESPAÑOL \\
\hline Bindung & Enlace \\
\hline Breite & Grosor \\
\hline Buchstabe & Letra, carácter \\
\hline Dehnung & Extensión \\
\hline Fuß & Pie \\
\hline Haar(strich) & Trazo fino, palo fino \\
\hline Kalligrafie & Caligrafía \\
\hline Kopf & Cabeza \\
\hline Kürzung & Acortamiento \\
\hline Ligatur & Ligado \\
\hline
\end{tabular}

\begin{tabular}{|c|c|} 
Proportionslehre & Norma de la proporción \\
\hline Rohrfederspitze & Punta del cálamo \\
\hline Rumpf & Cuerpo \\
\hline Schreibrohr & Cálamo \\
\hline Schrift & Caligrafía, escritura, alfabeto \\
\hline Schriftzug & Sentido del trazo \\
\hline Spitzen & Ángulos \\
\hline Strich & Trazo \\
\hline Taktstrich & Barra de compás \\
\hline
\end{tabular}

\section{A modo de conclusión}

Como se puede apreciar en nuestro trabajo, la traducción española del epílogo incluido por Schami en la novela original en alemán reviste las dificultades relacionadas con el lenguaje técnico de la caligrafía, insertado en un texto literario con un marcado carácter poético. Además de aptitudes literarias, el traductor precisa de conocimientos básicos en esta materia o, al menos, en la labor realizada por Ben Muqla en esta área de conocimiento. En este sentido, cabe destacar la utilidad de la metodología seguida, al complementar la traducción del alemán al español con la revisión por parte de una traductora especializada en lengua árabe. Con nuestro trabajo pretendemos mostrar no solo la traducción española del epílogo, sino también la necesidad que tiene el traductor de estar familiarizado con el campo específico de su traducción o de consultar a expertos en el mismo. El trabajo en equipos multidisciplinares se revela así como una valiosa aportación a la traducción editorial. Cabe añadir el interés de este tipo de textos sobre la lengua árabe en el contexto español, dado los lazos históricos y sociales de nuestro país con dicha lengua. Con nuestro estudio, esperamos haber contribuido a la recepción de Schami en el contexto hispanohablante y a la difusión de sus ideas sobre el alfabeto árabe y las posibles reformas del mismo.

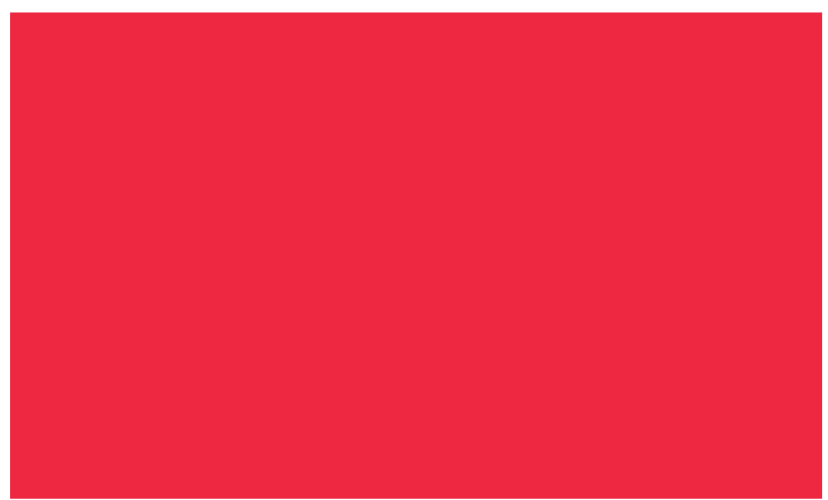


